( П ПЕТРОВА М. М., ШНАЙДЕР Н. А., ПРОНИНА Е. А., БОБРОВА О. П.

УДК 616.8-009.7-072.8

DOI: $10.20333 / 2500136-2020-3-61-69$

\title{
Диагностика нейропатической боли: шкалы и вопросники
}

М. М. Петрова ${ }^{1}$, Н. А. Шнайдер ${ }^{2}$, Е. А. Пронина ${ }^{1}$ О. П. Боброва ${ }^{1}$

${ }^{1}$ Красноярский государственный медицинский университет имени профессора В. Ф. Войно-Ясенецкого, Красноярск 660022, Российская Федерация ${ }^{2}$ Национальный медицинский исследовательский центр психиатрии и неврологии им. В. М. Бехтерева, Санкт-Петербург 192019, Российская Федерация

Резюме. Диагностика нейропатической боли может представлять трудности для специалистов первичного звена. Хроническая боль часто недооценивается и не поддается лечению. Разработаны несколько шкал и вопросников, которые оценивают наличие боли, интенсивность боли, распределение боли, а также психологическое и функциональное воздействие боли, включая оценку симптомов, имеющихся у пациента, а некоторые дополняются простыми методами клиническими обследованиями для выявления признаков поражения нервной системы.

Инструменты скрининга не идеальны, и было бы неправильно думать, что они могут заменить клиническую оценку. Однако наибольшая выгода будет заключаться в скрининговых целях, чтобы помочь специалисту в выявлении нейропатической боли или боли, которая имеет преимущественно нейропатический компонент.

Ключевые слова: нейропатическая боль, диагностика, скрининг, оценка боли, опросники, шкалы.

Конфликт интересов. Авторы декларируют отсутствие явных и потенциальных конфликтов интересов, связанных с публикацией настоящей статьи.

Для иитирования: Петрова ММ, Шнайдер НА, Пронина ЕА, Боброва ОП. Диагностика нейропатической боли: шкалы и вопросники. Сибирское медииинское обозрение. 2020;(3):61-69. DOI: 10.20333/2500136-2020-3-61-69

\section{Diagnosis of neuropathic pain: scales and questionnaires}

M. M. Petrova ${ }^{1}$, N. A. Shnayder ${ }^{1,2}$, E. A. Pronina ${ }^{1}$, O. P. Bobrova ${ }^{1}$

${ }^{1}$ Prof. V. F. Voino-Yasenetsky Krasnoyarsk State Medical University, Krasnoyarsk 660022, Russian Federation

${ }^{2}$ St. Petersburg V. M. Bekhterev Psychoneurological Research Institute, Saint Petersburg 192019, Russian Federation

Abstract. Diagnosis of neuropathic pain can be difficult for primary care professionals. Chronic pain is often underestimated and is not treatable. Several scales and questionnaires have been developed assessing pain presence, pain intensity, pain allocation, as well as psychological and functional effects of pain, including assessment of symptoms in a patient, and some being supplemented by simple methods of clinical examinations to detect signs of nervous system damage. Screening tools are not ideal, and it would be incorrect to think that they can replace clinical assessment. However, the greatest benefit will be for screening purposes to help a specialist identify neuropathic pain or pain that has predominantly neuropathic component.

Key words: neuropathic pain, diagnosis, screening, pain assessment, questionnaires, scales.

Conflict of interest. The authors declare the absence of obvious and potential conflicts of interest associated with the publication of this article.

Citation: Petrova MM, Shnayder NA, Pronina EA, Bobrova OP. Diagnosis of neuropathic pain: scales and questionnaires. Siberian Medical Review.2020;(3):61-69. DOI: 10.20333/2500136-2020-3-61-69

Болевые синдромы - состояния, которые достаточно широко распространены в практике врачей различных специальностей $[1,2]$. «Боль - это неприятное ощущение и эмоциональное переживание, связанное с действительным или возможным повреждением тканей или описываемое в терминах такого повреждения». Боль может быть острой и хронической. Как ответная сенсорная реакция на повреждение органов и тканей (болевое, ноцецептивное воздействие) возникает острая боль, которая может сопровождаться различными эмоциональными, вегетативными и другими проявлениями. Продолжительность острой боли зависит от времени заживления поврежденных органов и тканей, в том числе восстановления работы гладких мышц. Боль, которая остается после завершения обычного по длительности периода восстановления тканей организма расценивается как хроническая [3]. Согласно современным представлениям, на хронический характер боли указывает ее персиситирование в течение 3-х месяцев и более.

Также существует разделение боли наноцицептивную и нейропатическую. Ноцецепитвная боль обычно ограничена по времени, возникает в месте поражения, в ответ на различные травмирующие факторы, такие воспалительный процесс, операция, травматическое повреждение. К появлению нейропатической боли (НБ) приводит повреждение как периферического, так и центрального отдела соматосенсорной системы. Для нее характерно длительное изнурительное течение, отсутствие эффекта от простых и комбинированных анальгетиков, наличие положительных чувствительных феноменов, таких как парестезии, 
дизестезии, аллодиния, гипералгезия,в сочетании с негативными симптомами в виде гипестезии или анестезии. Повреждение периферической и центральной нервной системы не во всех случаях приводит к развитию НБ, необходимо ее сочетание с аномальной нейроанльной пластичностью, которая ведет к повышенной возбудимости ноцицептивной системы и снижению активности антиноцецептивной системы. По данным литературы, большой вклад в развитие нейропатического болевого синдрома вносит генетическая предрасположенность. Факторы, которые приводят к повреждению чувствительных афферентов ЦНС и ПНС очень разнообразны [4].

Этиологическая / анатомическая классификачия нейропатической боли (R. Baron et al., 2010) [5]

1. Периферические, очаговые или мультифокальные болезненные невропатии:

- постгерпетическая невралгия;

- боль после мастэктомии, боль после торакотамии;

- фантомные боли;

- невралгия тройничного нерва;

- хронические радикулопатии, синдром постсектэктомии;

- посттравматическая невропатия (синдром региональной невропатической боли);

- констрикционные синдромы;

- диабетическая мононевропатия;

- мормональная невралгия;

- ишемическая невропатия;

- синдром Баннварта (боррелиоз / болезнь Лайма);

- невралгическая амиотрофия плечевого сплетения;

- повреждение нервного сплетения, связанного с опухолью;

2. Периферические, генерализованные болезненные невропатии (полинейропатии):

- метаболические / пищевые (сахарный диабет, алкоголь, гипотиреоз, витаминная недостаточность);

- лекарственные (химиотерапевтические средства, включая препараты платины, таксоиды, винкристин, антиретровирусные препараты, другие препараты (дисульфирам, этамбутол, изониазид, нитрофурантоин, тиурацил, хлорамфеникол);

- инфекционные или постинфекционные, иммунологические (хроническая воспалительная полирадикулоневропатия: синдром Баннварта, Боррелиоз / болезнь Лайма), невропатия при ВИЧ-инфекции);

- наследственные (амилоидоз, болезнь Фабри, типы Шарко-Мари-Тута 2 типа, наследственные сенсорно-вегетативные невропатии типа 1 и 1B);
- токсические (акриламид, мышьяк, клиохинол, динитрофенол, этиленоксид, пентахлорфенол, таллий);

- злокачественные новообразования (паранеопластичесскаянейропатия).

3. Болевые нейропатии при поражении ЦНС:

- инсульт (особенно таламус или ствол мозга);

- повреждение спинного мозга;

- рассеянный склероз;

- сирингомиелия;

- смешанные болевые синдромы:

- хроническая боль в спине;

- боли, связанные с опухолью (инфильтрация);

- комплексный региональный болевой синдром (синдром Зудека, симпатическая рефлекторная дистрофия, каузалгия).

Выявление механизмов боли необходимо для определения лечебной тактики, поэтому в настоящее время большое внимание уделяется сопоставлению клинических проявлений с патогенетическими механизмами боли. Некоторые возможные механизмы представлены в таблице [6].

\section{Общие приниипы оценки нейропатической боли}

Пациенты с НБ часто встречаются не только в неврологической практике, но и могут оказаться на приеме у врача любой специальности[2]. Вместе с тем диагностика НБ не всегда является простой задачей. Разнообразная клиническая картина, субъективный характер ощущений, которые нельзя объективно измерить могут приводить к диагностическим сложностям. При оценке боли важная роль отводится самому пациенту. Объективная оценка боли - одна из сложных проблем в практике врачей различных специальностей [7]. У конкретного пациента восприятие боли может зависеть от многих причин, например, эмоциональный и физический статус человек в настоящий момент времени, этническая принадлежность,демографические факторы [8]. Ряд трудностей связан с тем, что зачастую оценка и интерпретация боли медицинским персоналом не совпадает с описанием своего состояния самим пациентом. С практической и научной точек зрения наибольший интерес представляют такие характеристики боли как ее интенсивность, влияние на различные аспекты и качество жизни пациента, эффект от лечения. Не меньшее значение имеют качественные характеристики боли и выявление именно неропатического болевого синдрома [7].

Сейчас для интерпретации болевых ощущений имеется ряд оригинальных англоязычных вопросников и шкал. Наиболее часто методики для оценки боли основываются именно на интерпретации 
Клиническая оценка и возможные механизмы симптомов при нейропатической боли

Clinical evaluation and possible mechanisms of neuropathic pain symptoms

\begin{tabular}{|c|c|c|c|}
\hline Симптом & Определение & Характеристика & Возможный механизм \\
\hline \multicolumn{4}{|c|}{ Спонтанная сенситизация или боль } \\
\hline Парестезия & $\begin{array}{c}\text { Неболевые патологические } \\
\text { ощущения }\end{array}$ & - & $\begin{array}{c}\text { Спонтанная активность } \\
\text { в низком плече A- } \beta \text { волокнах }\end{array}$ \\
\hline Дизастезия & $\begin{array}{c}\text { Неприятные патологические } \\
\text { неболевые ощущения }\end{array}$ & - & $\begin{array}{c}\text { Спонтанная активность } \\
\text { в C/A-ठ волокнах }\end{array}$ \\
\hline Пароксизмальная боль & $\begin{array}{c}\text { Короткий (секунды) приступ } \\
\text { стреляющей, пронизывающей боли } \\
\text { или боли, похожей на удар тока }\end{array}$ & - & $\begin{array}{l}\text { Спонтанная активность } \\
\text { С-ноцирецепторах }\end{array}$ \\
\hline Поверхностная боль & $\begin{array}{c}\text { Постоянная боль, локализованная } \\
\text { в коже, чаще жгучего характера }\end{array}$ & - & $\begin{array}{l}\text { Спонтанная активность } \\
\text { С-ноцирецепторов }\end{array}$ \\
\hline Глубокая боль & $\begin{array}{c}\text { Постоянная боль, локализованная } \\
\text { в мышцах, костях или внутренних } \\
\text { органах }\end{array}$ & - & $\begin{array}{c}\text { Спонтанная активность } \\
\text { ноцирецепторов мышц и суставов }\end{array}$ \\
\hline $\begin{array}{c}\text { Симпатическая } \\
\text { постоянная боль }\end{array}$ & & - & $\begin{array}{c}\text { Периферическая сенситизация: } \\
\text { симпатическая аффрерентная связь }\end{array}$ \\
\hline \multicolumn{4}{|c|}{ Вызванная боль } \\
\hline $\begin{array}{c}\text { Динамическая аллодиния, } \\
\text { провоцируемая механическим } \\
\text { раздражением }\end{array}$ & $\begin{array}{c}\text { Боль провоцируется обычными } \\
\text { неболевыми легкими } \\
\text { стимулирующими } \\
\text { движениями по коже }\end{array}$ & & $\begin{array}{c}\text { Центральная сенситизация: A- } \beta \\
\text { сенсорных волокон }\end{array}$ \\
\hline $\begin{array}{c}\text { Механическая } \\
\text { статическая гиперальгезия }\end{array}$ & $\begin{array}{c}\text { Боль провоцируется } \\
\text { мягким механическим } \\
\text { давлением на кожу }\end{array}$ & $\begin{array}{c}\text { Давящая боль, появляющиеся } \\
\text { зоне иннервации нерва }\end{array}$ & Периферическая сенситизация \\
\hline Механическая гиперальгезия & $\begin{array}{c}\text { Боль провоцируется колющими, } \\
\text { но неболевыми стимулами }\end{array}$ & $\begin{array}{c}\text { Резкая поверхностная боль, } \\
\text { локализованная в зоне первичного } \\
\text { воздействия и распространяющаяся } \\
\text { за пределы его }\end{array}$ & $\begin{array}{l}\text { Центральная сенситизация: } \\
\text { А-ठ сенсорных волокон }\end{array}$ \\
\hline $\begin{array}{l}\text { Временная суммация } \\
\text { (гиперстезия) }\end{array}$ & $\begin{array}{c}\text { Усиление боли при повторяющемся } \\
\text { воздействии одинаковых единичных } \\
\text { повреждающих стимулов }\end{array}$ & $\begin{array}{c}\text { Резкая поверхностная } \\
\text { боль увеличивающейся } \\
\text { интенсивности }\end{array}$ & $\begin{array}{l}\text { Центральная сенситизация: } \\
\text { А-б сенсорных волокон }\end{array}$ \\
\hline $\begin{array}{l}\text { Постсенсетизация } \\
\text { (гиперпатия) }\end{array}$ & $\begin{array}{c}\text { Боль появляется во время } \\
\text { воздействия и сохраняется } \\
\text { в течение некоторого времени } \\
\text { после прекращения стимуляции }\end{array}$ & Постоянная вызванная боль & Центральная сенситизация \\
\hline Холодовая гиперальгезия & $\begin{array}{c}\text { Боль провоцируется неболевыми } \\
\text { холодовыми стимулами }\end{array}$ & $\begin{array}{c}\text { Болезенные жгучие ощущения, } \\
\text { появляющиеся зоне иннервации } \\
\text { нерва }\end{array}$ & $\begin{array}{c}\text { Периферическая сенситизация } \\
\text { со снижением активности } \\
\text { холодового порога }\end{array}$ \\
\hline Тепловая гиперальгезия & $\begin{array}{c}\text { Боль провоцируется неболевыми } \\
\text { тепловыми стимулами }\end{array}$ & $\begin{array}{c}\text { Болезенные жгучие ощущения, } \\
\text { появляющиеся в зоне иннервации } \\
\text { нерва }\end{array}$ & $\begin{array}{c}\text { Периферическая сенситизация } \\
\text { со снижением активности } \\
\text { теплового порога }\end{array}$ \\
\hline
\end{tabular}

утверждений пациентов. Использование специализированных шкал для оценки боли помогает достаточно быстро собрать полную информацию о болевых ощущениях индивида. Применение вопросников также позволяет сопоставлять результаты лечения, выявлять группы риска и правильно подбирать пациентов. Подбор анкет для оценки боли зависит от тех целей, которые ставит перед собой врач или исследователь.

\section{Шкалы и вопросники \\ для оценки интенсивности боли}

На каждом этапе лечения пациента большое значение имеет оценка интенсивности болевого синдрома. Это необходимо, в том числе чтобы определить эффективность проводимой болеутоляющего терапии.

В настоящее время широко используются «аналоговые шкалы боли», в основе которых лежит выявление связи между выраженностью боли и длиной отрезка, указанного пациентом между точками «боль отсутствует» и «невыносимая или максимальная боль». Визуальная аналоговая шкала - ВАШ (Visual Analogue Scale, VAS, E. C. Huskisson, 1974, англ.). В основном, применяется линейка из бумаги, картона или пластика длиной $10 \mathrm{~cm}$. С обратной стороны линейки нанесены сантиметровые деления, по которым медицинский персонал отмечает полученное значение и заносит в лист наблюдения. К достоинствам этой шкалы можно отнести ее простоту и удобство. Однако ВАШ является одномерной шкалой и позволяет оценить только интенсивность боли [7]. Она также подходит для динамической оценки боли при подборе обезболивающей терапии. Повторные измерения интенсивности боли с помощью этой шкалы позволяют оценить эффективность лечения. Изменение выраженности боли можно считать объективным и значимым, если каждое последующее значение ВАШ 
(на неградуированной линейке длиной $10 \mathrm{~cm}$ ) более чем на 13 мм отличается от предыдущего. У пациентов с острой болью в послеоперационном периоде, онкологических больных, по мнению других авторов, на достаточную эффективность лечения указывает уменьшение интенсивности боли на 30-35 \%. Обычно ни дети (в возрасте 5 лет и старше), ни взрослые не испытывают трудностей при использовании этой шкалы. Исключение составляют больные со сниженным зрением и когнитивной дисфункцией, чаще это пациенты старшей возрастной группы [7].

Следующий доступный метод оценки интенсивности боли - цифровая рейтинговая шкала, ЦРШ (Numerical Rating Scale, NRS). Для измерения выраженности болевых ощущений пациент на градуированной от 1 до 100 шкале отмечает число, которое по его ощущениям наиболее точно отражает силу, испытываемой им боли. Полученные результаты авторы предлагают интерпретировать следующим образом: значения от 0 до 0.5 балла говорят об отсутствии боли, от 1 до 4 баллов - слабая боль (по цифровой оценочной шкале от 0 до 10 баллов), от 5 до 6 баллов умеренная боль, от 7 до 10 баллов - сильная боль. К преимуществам этой шкалы можно отнести то, что анкетирование можно проводить дистанционно, например, по телефону. Ее можно использовать у пациентов с плохим зрением, у людей, которые в силу разных причин не могут самостоятельно пользоваться ручкой и бумагой.

Вербальная описательная шкала Verbal Descriptor Scale (F. Gaston-Johansson, M. Albert, E. Fagan et al., 1990) позволяет оценить наличие боли у пациента в настоящий момент времени. Если боли нет, то его состояние оценивается в 0 баллов. Если наблюдаются болевые ощущения, необходимо спросить: «Вы могли бы сказать, что боль усилилась, или боль невообразимая, или это самая сильная боль, которую вы когда-либо испытывали?» Если это так, то фиксируется самая высокая оценка в 10 баллов. Если же нет ни первого, ни второго варианта, то далее необходимо уточнить: «Можете ли вы сказать, что ваша боль слабая, средняя (умеренная, терпимая, несильная), сильная (резкая) или очень (особо, чрезмерно) сильная (острая)». Таким образом, возможны шесть вариантов оценки боли: 0 - нет боли; 2 - слабая боль; 4 - умеренная боль; 6 - сильная боль; 8 - очень сильная боль; 10 - нестерпимая боль. Использование этой шкалы возможно у детей старше 7 лет, а также у взрослых пациентов с нетяжелыми когнитивными нарушениями, для оценки и хронической и острой боли [9]. Боль является индивидуальным опытом, возможно поэтому именно словесное описание, может помочь пациенту более точно оценить интенсивность боли.
Из тестов, позволяющих оценить интенсивность боли, в рутинной практике наибольшее распространение получила ВАШ.

Для детей общепринятой является Лицевая шкала боли (Faces Pain Scale, D. Bieri et al., 1990) [9]. Шкала состоит из семи нарисованных лиц. На первой картинке лицо имеет нейтральное выражение, изображения следующих лиц показывают нарастание боли. Ребенку необходимо показать то лицо, которое, по его ощущениям, лучше всего передает уровень, испытываемой им боли. Таким образом, возможны шесть вариантов оценки боли: (оценка теста показана слева направо: 0, 2, 4, 6, 8, 10 баллов), где 0 баллов - «совсем не больно», 10 баллов - «очень-очень больно». Возможно применение модифицированной лицевой шкалы боли (The Faces Pain Scale-Revised, FPS-R, англ.), предложенной впервые C. L. Von Baeyer et al. $[10,11]$. В данном варианте также остается изображение с нейтральным выражением лица, однако общее число использованных картинок сократили до шести, каждая из которых имеет цифровую оценку в диапазоне от 0 до 10 баллов.

Нужно отметить, что для оценки НБ использование аналоговых шкал будет недостаточным, поскольку они описывают лишь интенсивность боли.

Вопросник боли Мак-Гилла (McGill Pain Questionnaire, MPQ, англ.) разработан в 1975 году профессором Ronald Melzack в Монреале (Канада) в университете McGill. «Он включает в себя сенсорную, эмоциональную и оценочную составляющие, которые согласуются со специализированными системами в мозге: сенсорно-дискриминативное измерение боли определяется первичными быстрыми проводящими волокнами спинного мозга; мощная мотивационная и неприятная аффективная характеристики согласуются с деятельностью ретикулярной формации и лимбическими структурами, которые находятся под влиянием медленных демиелинизированных С-волокон спинного мозга; неокортекс и высшие центры регуляции центральной нервной системы, определяющие боль с точки зрения прошлого опыта, управляют познавательно-оценочной составляющей» [12].

Лист вопросника включает 78 пунктов, которые предназначены для описания боли, разделенных по 20 кластерам, которые отражают 3 основных болевых аспекта (сенсорный, эмоциональный и оценочный) и 1 смешанный фактор. Пациенты отмечают слова, которые наиболее точно соответствуют их болевым ощущениям (не обязательно в каждом кластере). Нужно выбрать только одно слова из кластера. Сенсорный аспект - Sensory Pain Rating (SPR) - определяется 1-10-м кластерами, эмоциональный - Affective Pain Rating (APR) -11-15-м кластерами, оценочный Evaluative Pain Rating (EPR) - 16-м кластером. 17-20-й 
подклассы отражают другое разнообразие болевого синдрома - Miscellaneous Pain Rating (MPR). Все факторы и кластеры ранжированы по баллам, которые увеличиваются по мере выраженности боли. Каждый кластер включает от 2 до 5 определений боли, описывающих уровень интенсивности боли в каждом кластере. Итоговое значение (ранговый индекс боли - Pain Rating Index, PRI, англ.) получается в результате суммирования всех выбранных слов из всех 20 кластеров и варьирует от 0 до 78 баллов. Итоговые суммы могут быть также подсчитаны для каждого фактора сложением значений дескрипторов, соответствующих фактору подклассов. Сумма балов для сенсорного фактора варьирует от 0 до 42, для эмоционального - от 0 до 14, для оценочного - от 0 до 5, а для MPR - от 0 до 20.Текущее ощущение интенсивности боли определяется по шкале от 0 до 5 баллов. Полученные результаты не являются параметрическими величинами, но поддаются статистической обработке. Данные, полученные в ходе анкетирования могут служить для оценки не только боли, но и эмоционального состояния пациента. В клинических исследованиях вопросник боли Мак-Гилла используется уже многие годы. Однако, учитывая объемность MPQ и сложность заполнения, этот вопросник не нашел широкого применения в практическом здравоохранении $[7,13,14]$.

Для использования в обычной клинической практике разработана Краткая форма вопросника боли Мак-Гилла (Short Form McGill Pain Questionnaire, SF-MPQ, англ.). Она предназначена для оценки, как острой, так и хронической боли [15]. SF-MPQ состоит из 15 пунктов (11 сенсорных и 4 аффективных), взятых из оригинального MPQ. Каждое выбранное слово оценивается от 0 баллов (нет боли) до 3 баллов (сильная боль). Итоговое значение (ранговый индекс боли - Pain Rating Index, PRI, англ.) высчитывается путем суммирования баллов (диапазон 0-45 баллов). Пороговых значений для этой шкалы не выведено. Высокий балл, указывает на более сильную боль.

Краткий вопросник боли (Brief Pain Inventory, BPI, C. Cleeland et al., 1994, англ.), позволяет оценить выраженность боли и ее влияния на основные аспекты качества жизни пациентов - ежедневную активность, способность к передвижению, возможность выполнять домашние дела, отношения с другими людьми, настроение, сон, и способность получать удовольствие [16]. Он может быть заполнен самим пациентом, врачом в ходе беседы или в форме телефонного опроса. BPI также позволяет оценить изменение интенсивности боли в течение суток, эффективность медикаментозных средств [17]. К достоинствам ВРI можно отнести: краткость и простоту заполнения, возможность применения в повседневной работе с тяжелыми пациентами [7]. Модифицированный вариант BPI использовался для оценки тяжести симптомов боли у пациентов с диабетической полинейропатией [18].

\section{Шкалы и вопросники для дифберениированной диагностика нейропатической боли}

Диагностика нейропатического компонента боли базируется на выявлении различных симптомов, характерных для НБ и отражающих различные механизмы ее формирования [19-27].

В повседневной клинической практике для быстрого разделения НБ и боли, не имеющей нейропатического характера, было предложено несколько методик, основанных на описательных определениях боли. Целью применения скрининговых шкал является насторожить врача в отношении возможного невропатического механизма боли, особенно при первичном обращении пациента, но они не заменяют клинический диагноз [28-35].

Для упрощения выявления НБ в настоящее время могут быть использованы следующие скрининговые шкалы: Leeds Assessmentof Neuropathic Symptoms and Signs (LANSS) [36], self-report LANSS (S-LANSS) [37, 38], Douleur Neuropathic 4 Questions (DN4) [39], PainDETECT [40], Neuropathic Pain Questionnaire (NPQ) [41], ID Pain [42] и др. Определение нейропатического компонента болевого синдрома влияет на тактику ведения пациента и выбор лекарственных препаратов, поэтому диагностические шкалы должны быть в достаточной мере надежными и точными. Ряд шкал может быть рекомендован для оценки эффективности лечения нейропатических симптомов в том числе в клинических исследованиях для оценки результатов лечения и лучшего определения профиля респондента.

Лидская шкала оценки нейропатических симптомов (Leeds Assesment of Neuropathic Symptoms and Signs, LANSS, Bennett, 2001, англ.) содержит 5 пунктов, описывающих симптомы, и 2 - относящиеся к клиническим обследованиям. По сравнению с клиническим диагнозом его чувствительность и специфичность составляют 82-91 \% и 80-94 \% соответственно. Опросник LANSS заполняет клиницист, который проводит опрос и объективное обследование больного. При этом врач обращает внимание на выраженность дизестезии, аллодинии, а также о наличии острой пароксизмальной боли и вегетативной дисфункции. Заполнение данного опросника не требует больших затрат времени, поэтому его можно рекомендовать для ежедневного использования. Полученная в итоге сумма больше 12 баллов, говорит о том, что у больного имеет место нейропатический компонент формирования боли $[36,43]$. 
Применение опросника в ряде клинических испытаний показало, что, LANSS обладает высокой чувствительностью и специфичностью как для дифференциальной диагностики нейропатической и ноцицептивной боли, так и для определения эффективности различных лечебных мероприятий. Хотя изначально шкала не предполагала количественной оценки интенсивности боли $[44,45,46]$.

Версия вопросника LANSS для самостоятельного заполнения пациентом (Self-complete Leeds Assessment of Neuropathic Symptoms and Signs, SELFLANSS, S-LANSS, англ.) была разработана 2005 году M. Bennett. Хотя чувствительность и специфичность S-LANSS несколько меньше, чем у ее полного варианта, эта шкалаимеет достаточно высокую диагностическуюзначимость: S-LANSS правильно определяет тип боли в $75 \%$ случаев [37,38].

Вопросник ДН4 (Douleur Neuropathiqueen 4 questions, DN4, англ.) разработан Boushassira и соавторами в 2005 году во Франции (The French Neuropathic Pain Group - FNPG, англ.) и буквально означает 4 вопроса о НБ. Вопросник содержит 10 позиций, из которых: 7 связаны с качественными характеристиками боли (жжение, ощущение холода, удар электрического тока), 3 позиции относятся к клиническому обследованию пациента и направлены на выявление гипоэстезии, гиперэстезии и аллодинии [35]. Для подтверждения нейропатического характера боли, необходимо получить 4 положительных ответа из 10. Чувствительность методики составляет до 86 \%, специфичность - 90 \%. Вопросник применяли в эпидемиологических исследованиях населения, в том числе у пациентов с сахарным диабетом [47]. Вопросник ДН4 прост в заполнении и интерпретации результатов. В России он используется наиболее часто [2,20].

Вопросник боли Pain DETECT был разработан и предварительно валидизирован для пациентов с болью в спине. Pain DETECT включает в себя 9 пунктов, в том числе, картинку-схему человеческой фигуры для указания заинтересованных мест, шкалу ВАШ и опросник, направленный на выявление спонтанных и вызванных симптомов НБ [40]. Семь пунктов оценивают интенсивность сенсорных ощущений в диапазоне от «совсем нет» до «очень сильно», 2 пункта при помощи рисунка оценивают иррадиацию болей и временные характеристики индивидуального болевого паттерна. Выраженность каждого симптома оценивается в баллах, затем высчитывается общий балл. Более 19 баллов означает вероятное наличие у пациента нейропатического компонента боли, от 13 до 18 баллов - показания неоднозначны, однако болевой компонент может иметь место. Диагностическая ценность вопросника составляет $3 \%$.

Boпросник ID-pain разработан в США в 2006 году в большей степени для скрининга нейропатического компонента боли и не требует клинического обследования. Состоит из 5 описательных позиций и 1 вопроса относительно того, локализуется ли боль в суставах для выявления ноцицептивного компонента. Пороговым значением, указывающим на нейропатический характер боли, является 3 балла и более. Проведенные исследования показали невысокую чувствительность и специфичность данной методики [42].

Шкала нейропатической боли, специально разработана для оценки НБ учеными из Вашингтонского медицинского университета Bradley Galer и Mark Jensen. Шкала Нейропатической Боли, ШНБ (Neuropathic Pain Scale, NPS, англ.) [48] оценивает ощущения больного по десяти пунктам: первые два из них характеризуют интенсивность боли (практически совпадающая с ВАШ) и дискомфорт; следующие восемь пунктов оценивают специфические качества боли: колющая, жгучая, тупая, холодная, ноющая, зудящая, глубокая, поверхностная. При этом каждый пункт имеет 10 числовых/словесных градаций. Есть графа для обозначения изменения болевых ощущений во времени. Вопросник NPS позволяет проводить качественную оценку вклада различных симптомов в клиническую картину боли, поэтому при его анализе, баллы не суммируются $[43,49,50]$. Вопросник подходит при дифференциальной диагностики различных нейропатических болевых синдромов [51]. Имеется опыт использования данной шкалы для оценки эффективности лечения НБ. Однако ей недостает нескольких болевых характеристик, обычно наблюдаемых при НБ, полную валидизацию она прошла только для рассеянного склероза [52].

Вопросник симптомов невропатической боли (Neuropathic Pain Symptom Inventory, NPSI, англ.) разработан во Франции и Бельгии в 2004 году. NPSI включает в себя 12 пунктов; 10 из них предназначены для выявления симптомов, характерных для НБ, и 2 пункта нужны, что бы оценить длительность спонтанной постоянной и пароксизмальной боли. Итоговая сумма баллов, набранных по всем 10 пунктам, позволяет оценить общую выраженность болевого синдрома. Увеличение или уменьшение общего балла NPSI связаны с изменениями болевого синдрома пациента [53].

NPS и NPSI позволяют оценить выраженность симптомов у пациентов с НБ, определить эффективность различных методов лечения, а также помочь выяснить механизм(ы), эффективность различных групп лечения [17]. NPS и NPSI не дифференцируют пациентов с НБ от пациентов с ноцецептивной болью.

\section{Заключение}

Наличие простых и общепринятых инструментов оценки болевого синдрома необходимо не только в повседневной работе врача, но и при проведении научных исследований. 
Единый подход к использованию различных вопросников и шкал, позволил бы стандартизировать и унифицировать изучение различных аспектов данной проблемы, сопоставлять и сравнивать результаты терапии в разных клиниках и центрах, упрощая профессиональное общение специалистов.

Детальный клинический осмотр является обязательным при обследовании пациента с болевым синдромом. В то время как вопросники и шкалы являются удобным вспомогательным методом диагностики боли [1].

\section{Литература / References}

1. Данилов АБ, Давыдов ОС. Диагностика и фармакотерапия нейропатической боли. Русский медииинский журнал. 2009; 17(20):1389-1394. [Danilov AB, Davydov OS. Diagnostics and pharmacology of neuropathic pain. Russian Medical Journal. 2009;17(20):1389-94. (In Russian)]

2. Яхно НН, Кукушкин МЛ, Данилов АБ, Амелин AВ, Давыдов ОС, Куликов СМ. Результаты Российского эпидемиологического исследования распространенности нейропатической боли, ее причины и характеристики в популяции амбулаторных больных, обратившихся к врачу неврологу. Боль. 2008;3(20):24-32. [Jahno NN, Kukushkin ML, Danilov AB, Amelin AV, Davydov OS, Kulikov SM. Results of the Russian epidemiological study of the prevalence of neuropathic pain, its causes and characteristics in a population of outpatients who consulted a neurologist. Pain. 2008;3(20):24-32. (In Russian)]

3. PainIAft So. IASP Taxonomy - IASP. 2015. Accessed 28 May, 2020. http://www.iasp-pain.org/ Education/Content. aspx?ItemNumber $=1698 \&$ navitemNumber $=576$

4. Баринов АН, Ахмеджанова ЛТ, Махинов КА. Алгоритмы диагностики и лечения невропатической боли при поражении периферической нервной системы. Русский медицинский журнал. 2016;24(3):154-162. [Barinov AN, Ahmedjanova LT, Makhinov KA. Algorithms for diagnosis and treatment of neuropathic pain in the defeat of the peripheral nervous system. Russian Medical Journal. 2016; 24 (3): 154-162. (In Russian)

5. Baron R, Binder A, Wasner G. Neuropathic pain: diagnosis, pathophysiological mechanisms, and treatment. Lancet Neurology. 2010;9(8):807-19. DOI: 10.1016/S14744422(10)70143-5

6. Mindruta I, Cobzaru AM, Bajenaru O. Overview of Neuropathic Pain Diagnosis and Assessment. An Approach Based on Mechanisms, Neuropathic Pain. PainIAftSo. IASP Taxonomy - IASP. 2015. Accessed 28 June, 2020. http:// www.iasp-pain.org/Education/Content.aspx?ItemNumber $=1698$ \&navitemNumber $=576$

7. Харченко ЮА. Адекватная оценка боли - залог её успешного лечения. Universum: Медицина и фармакология: электронный научный журнал. 2014;5(4). Ссылка активна на 28.05.2020. [Kharchenko YA.
Adequate assessment of pain is the pledge of successful treatment. Scientific Journal Universum: Medicine and Pharmacology. 2014;5(4). Accessed 28 May, 2020. (In Russian)] http://7universum.com/ru/med/archive/item/1229

8. Yang JC, Clark WC, Tsui SL, Ng KF, Clark SB. Preoperative multidimensional affect and pain survey (MAPS) scores predict postcolectomy analgesia requirement. The Clinical Journalof Pain. 2000;16(4):314-320. DOI: org/10.1097/00002508-200012000-00007

9. Bieri D, Reeve RA, Champion GD, Addicoat L, Ziegler JB. The Faces Pain Scale for the self-assessment of the severity of pain experienced by children: development, initial validation, and preliminary investigation for ratio scale properties. Pain. 1990;41(2):139-50. DOI: org/10.1016/0304-3959(90)90018-9

10. Tsze DS, Hirschfeld G, von Baeyer CL, Bulloch B, Dayan PS. Clinically significant differences in acute pain measured on self-report pain scales in children. Academic emergency medicine: official journal of the Society for Academic Emergency Medicine.2015;22(4):415-422. DOI:10.1111/acem.12620

11. Von Baeyer CL. Children's self-reports of pain intensity: Scale selection, limitations and interpretation. Pain Research and Management: The Journal of the Canadian Pain Society.2006;11(3):157-162.

12. Melzack R. The McGill Pain Questionnaire: major properties and scoring methods. Pain. 1975;(1):277-299. DOI:org/10.1016/0304-3959(75)90044-5

13. Melzack R. The McGill pain questionnaire: from description to measurement. Anesthesiology. 2005;103(1): 199-202. DOI: org/10.1016/0304-3959(87)91074-8

14. Кастыро ИВ, Попадюк ВИ, Благонравов МЛ, Ключникова ОС, Кравцова ЖВ. Опросник боли МакГилла как метод определения уровня болевого синдрома у пациентов после риносептопластики и полипотомии носа. Бюллетень Восточно-Сибирского научного иентра Сибирского отделения Российской Академии медицинских наук. 2012;86(4-2):68-71. [Kastyro IV, Popadyu VI, Blagonravov ML, Kluchnikov OS, Kravtsova ZhV. McGill pain questionnaire as a method of identification of pain syndrome level in patients after rhynoseptoplasty and polypothomy. Bulletin of the East Siberian Scientific Center SBRAMS. 2012;86(4-2):68-71. (In Russian)]

15. Melzack R. The Short-Form McGill Pain Questionnaire. Pain. 1987;30(2):191-197. DOI: org/10.1016/03043959(87)91074-8

16. Cleeland CS, Ryan KM. Pain assessment: global use of the Brief Pain Inventory. Annals of the Academy of Medicine, Singapore. 1994;23(2):129-38.

17. Semenchuk MR, Sherman. Effectivness of tizanidine in neuropathic pain: an open-label study. Pain. 2000; (1):285-292. DOI: 10.1054/jpai.2000.9435

18. Zelman DC, Gore M, Dukes E, Tai KS, Brandenburg N. Validation of a modified version of the brief pain inventory for painful diabetic peripheral neuropathy. 
Journal of Pain and Symptom Management. 2005;29(4): 401-410. DOI: 10.1016/j.jpainsymman.2004.06.018

19. Баринов АН, Мурашко НК, Терентьева НВ, Яворский ВВ. Невропатическая боль при поражении периферической нервной системы: стратификация лечения. Медииинский совет. 2013;(4):54-63. [Barinov AN, Murashko NK, Terent'eva NV, Javorskij VV. Neuropathic pain at peripheral nervous system impairment: stratifi cation of treatment. Medicinskij Sovet. 2013;(4):54-63. (In Russian)]

20. Быков ЮН, Калягин АН. Дифференциальная диагностика нейропатической боли. Неврология, нейропсихиатрия, психосоматика. 2015;(1):4-10. [Bykov JuN, Kaljagin AN. Differentiated diagnostics of neuropathic pain. Neurology, neuropsychiatry, psychosomatics. 2015;(1):4-10. (In Russian)] DOI: http://dx.doi. org/10.14412/2074-2711-2015-1-4-10

21. Быков ЮН, Левина ГЮ, Борисов АС. Диагностика и лечение нейропатической боли при заболеваниях периферической нервной системы. Сибирский медицинский журнал (Иркутск). 2014;131(8):6-30.[Bykov YN, Levina GY, Borisov AS. Diagnostics and treatment of neuropathic pain in patients with perypheral nervous system diseases. Siberian Medical Journal (Irkutsk). 2014;131(8): 6-30. (In Russian)]

22. Левина ГЮ, Быков ЮН, Борисов АС. Диагностика и лечение периферической нейропатической боли. Бюллетень Восточно-Сибирского научного центра Сибирского отделения Российской Академии медииинских наук.2015;101(1):72-76. [Levina GY, Bykov YN, Borisov AS. Diagnostics and therapy of peripheral neuropathy. Bulletin of the East Siberian Scientific Center SBRAMS. 2015;101(1):72-76. (In Russian)

23. Мурашко НК. Нейропатическая боль: междисциплинарный подход в диагностике и лечении. Рекомендации 2013. Ссылка активна на 28.05.2020. [Murashko NK. Neuropathic pain: interdisciplinary approaches in diagnostics and treatment. Guideline of EFNS. 2013. Accessed 28 May, 2020. (In Russian)] http:// www.health-medix.com/articles/misteztvo/2013-07-24/ neuropat_bol.pdf

24. Бывальцев ВА, Белых ЕГ, Алексеева НВ, Сороковиков ВА. Применение шкал и анкет в обследовании пациентов с дегенеративным поражением поясничного отдела позвоночника: метод. рекомендации. Иркутск: ФГБУ НЦРВХ СО РАМН; 2013. 32 c. [Byvaltsev VA, Belykh EG, Alekseeva NV, Sorokovikov VA. Scale and questionnaire in study of patients with low back disorders: methodical guideline, Irkutsk: FGBU NTSRVH SO RAMN;2013. 32p. (In Russian)]

25. Яхно НН, Строков ИА, Новосадова МВ, Баринов АН. Клинические методы оценки тяжести диабетической полиневропатии. Неврологический журнал. 2000;(5):14-19. [Yakhno NN, Strokov IA, Novosadov MV, Barinov AN. Clinical methods for assessing the severity of diabetic polyneuropathy. The Neurological Journal. 2000;(5):14-19. (In Russian)]

26. Халикова ЕЮ. Нейропатическая боль как компонент острой и хронической постоперационной и посттравматической боли: от диагностики к рациональной фармакотерапии. Русский медицинский журнал.2014;22(32):38-42. [Halikova EJu. Neuropathic pain as a component of acute and chronic post-operation and post-traumatic pain: from diagnostics to rational pharmacotherapy. Russian Medical Journal. 2014;22(32):38-42. (In Russian)]

27. Овсянников ВГ, Бойченко АЕ, Алексеев ВВ, Алексеева НС. Инициальные механизмы формирования боли. Журнал фундаментальной медицины и биологии. 2015;(3):4-12. [Ovsyannikov VG, Boichenko AE, Alekseev VV, Alekseeva NS. The initial formation and mechanisms of the pain.Fundamental Medicine and Biology.2015;(3):4-12. (In Russian)]

28. Bennett MI, Attal N, Backonja MM, Baron R, Bouhassira D, Freynhagen R, Scholz J, Tölle TR, Wittchen HU, Jensen TS. Using screening tools to identify neuropathic pain.Pain. 2007;127;(3):199-203. DOI: 10.1016/j. pain.2006.10.034

29. Breivik H, Borchgrevink PC, Allen SM, Rosseland LA, Romundstad L, Hals EK, Kvarstein G, Stubhaug A. Assessment of pain. British Journal of Anaesthesia. 2008;101(1):17-24. DOI: 10.1093/bja/aen103

30. Dansie EJ, Turk DC, Colvin L. Rowbotham DJ. Assessment of patients with chronic pain. British Journal of Anaesthesia. 2013;111(1):19-25. DOI:10.1093/bja/aet124

31. Haanpaa ML, Backonja MM, Bennett MI, Bouhassira D, Cruccu G, Hansson PT, Jensen TS, Kauppila T, Rice AS, Smith BH, Treede RD, Baron R. Assessment of neuropathic pain in primary care. The American Journal of Medicine. 2009;122(10):13-22. DOI: 10.1016/j.amjmed. 2009.04.006

32. Hansson P, Hanpä M. Diagnostic work-up of neuropathic pain: computing, using questionnaires or examining the patient? European Journal of Pain (London, England). 2007;11(4): 367-369. DOI: 10.1016/j.ejpain.2006.12.005

33. Marchettini P. Editorial-The burning case of neuropathic pain wording. Pain. 2005;(114):313-314. DOI: org/10.1016/j.pain.2005.01.016

34. May S, Serpell M. Diagnosis and assessment of neuropathic pain. F1000 Medicine Reports.2009;(1):76. DOI: $10.3410 / \mathrm{M} 1-76$

35. Bennett M. The LANSS Pain Scale the Leeds Assessment of Neuropathic Symptoms and Signs. Pain.2001; (92):147-157. DOI:org/10.1016/s0304-3959(00)00482-6

36. Bennett MI, Smith BH, Torrance N, Potter J. The S-LANSS score for identifying pain of predominantly neuropathic origin: validation for use in clinical and postal research. Pain. 2005;(6):149-158. DOI: 10.1016/j. jpain.2004.11.007 
37. Weingarten TN, Watson JC, Hooten WM. Validation of the S-LANSS in the community setting. Pain. 2007;132(1-2):189-194. DOI: 10.1016/j.pain.2007.07.030

38. Bouhassira D, Attal N, Alchaar H, Boureau F, Brochet B, Bruxelle J, Cunin G, Fermanian J, Ginies P, Grun-Overdyking A, Jafari-Schluep H, Lantéri-Minet $\mathrm{M}$, Laurent B, Mick G, Serrie A, Valade D, Vicaut E. Comparison of pain syndromes associated with nervous or somatic lesions and development of a new neuropathic pain diagnostic questionnaire (DN4). Pain.2005;(114):29-36. DOI: $10.1016 /$ j.pain.2004.12.010

39. Freynhagen R, Baron R, Gockel U, Tolle T. Pain DETECT: a new screening questionnaire to detect neuropathic components in patients with back pain. Current Medical Research and Opinion. 2006;(22):1911-1920. DOI: 10.1185/030079906X132488

40. Backonja MM, Krause SJ. Neuropathic Pain Questionnaire - Short Form. The Clinical Journal of Pain. 2003;(19):315-316. DOI:10.1097/00002508-20030900000005

41. Portenoy R. Development and testing of a neuropathic pain screening questionnaire: ID Pain. Current Medical Research and Opinion. 2006;22(8):1555-65. DOI: 10.1185/030079906X115702

42. Данилов АБ, Давыдов ОС. Диагностические шкалы для оценки невропатической боли. Боль. 2007;16(3):11-15. [Danilov AB, Davydov OS. Diagnostic scales for assessing neuropathic pain. Pain. 2007;16(3): 11-15. (In Russian)]

43. Potter J, Higginson IJ, Scadding JW, Quigley C. Identifying neuropathic pain in patients with head and neck cancer: use of the Leeds Assessment of Neuropathic Symptoms and Signs Scale. Journal of the Royal Society of Medicine. 2003;96(8):379-383. DOI: org/10.1258/jrsm.96.8.379

44. Yawn BP, Wollan PC, Weingarten TN, Watson JC, Hooten WM, Melton LJ. The prevalence of neuropathic pain: clinical evaluation compared with screening tools in a community population. Pain Medicine (Malden, Mass.).2009;10(3):586-593. DOI: $10.1111 / \mathrm{j} .1526-4637$. 2009.00588.x

45. Yucel A, Senocak M, Kocasoy Orhan E, Cimen A, Ertas M. Results of the Leeds Assessment of Neuropathic Symptoms and Signs Pain Scale in Turkey: a validation study. Pain. 2004;5(8):427-432. DOI: 10.1016/j.jpain.2004.07.001

46. Mathieson S, Maher CG, Terwee CB, Folly de Campos T, Lin CW. Neuropathic pain screening questionnaires have limited measurement properties. A systematic review.Journal of Clinical Epidemiology. 2015;68(8):957-66. DOI: 10.1016/j.jclinepi.2015.03.010

47. Galer BS, Jensen MP. Development and preliminary validation of a painmeasure specific to neuropathic pain: the Neuropathic Pain Scale. Neurology. 1997;(48):332-338. DOI: org/10.1212/wnl.48.2.332
48. Jensen MP, Dworkin RH, Gammaitoni AR, Olaleye DO, Oleka N, Galer BS. Assessment of pain quality in chronic neuropathic and nociceptive pain clinical trials with the Neuropathic Pain Scale.Pain. 2005;(6):98-106. DOI: $10.1016 /$ j.jpain.2004.11.002

49. Krause SJ, Backonja MM. Development of a Neuropathic Pain Questionnaire. The Clinical Journal of Pain. 2003;(19):306-314. DOI: org/10.1097/00002508200309000-00004

50. Fishbain DA, Lewis JE, Cutler R, Cole B, Rosomoff HL, Rosomoff RS. Can the neuropathic pain scale discriminate between non-neuropathic and neuropathic pain? Painmedicine (Malden, Mass.).2008;9(2):149-160. DOI: $10.1111 /$ j.1526-4637.2007.00302.x

51. Rog DJ, Nurmikko TJ, Friede T, Young CA. Validation and reliability of the Neuropathic Pain Scale (NPS) in multiple sclerosis. The Clinical Journal of Pain. 2007;23(6):473-481. DOI: 10.1097/AJP.0b013e31805d0c5d

52. Zelman DC, Gore M, Dukes E, Tai KS, Brandenburg N. Validation of a modified version of the brief pain inventory for painful diabetic peripheral neuropathy. Journal of Pain and Symptom Management. 2005;29(4): 401-410. DOI: 10.1016/j.jpainsymman.2004.06.018

\section{Сведения об авторах}

Петрова Марина Михайловна, д.м.н., профессор, Красноярский государственный медииинский университет имени профессора В.Ф. Войно-Ясенеикого; адрес: Российская Федераиия, 660022, г. Красноярск, ул. Партизана Железняка, д. 1; тел.: +7(391)2280628; e-mail: stk99@yandex.ru, http://orcid.org/0000-0002-8493-0058

Шнайдер Наталья Алексеевна, д.м.н., профессор, Национальный медииинский исследовательский иентр психиатрии и неврологии им. В.М. Бехтерева; адрес: Российская Федераиия, 192019, Санкт-Петербург, ул. Бехтерева, 3; Красноярский государственный медицинский университет имени профессора В.Ф. Войно-Ясенеикого; адрес: Российская Федераи,ия, 660022, г. Красноярск, ул. Партизана Железняка, д. 1; тел.: +7(391)2280628; e-mail: naschnaider@yandex.ru, http://orcid.org/0000-0002-2840-837X

Пронина Елена Александровна, к.м.н., доцент, Красноярский государственный медииинский университет имени профессора В.Ф. Войно-Ясенеикого; адрес: Российская Федераиия, 660022, г. Красноярск, ул. Партизана Железняка, д. 1; тел.: +7(391)2280628; e-mail: diinny@mail.ru, http://orcid.org/0000-0002-8269-6317

Боброва Ольга Петровна, к.м.н., доцент, Красноярский государственный медииинский университет имени профессора В.Ф. Войно-Ясенеикого; адрес: Российская Федеращия, 660022, г. Красноярск, ул. Партизана Железняка, д. 1; тел.: +7(391)2280628; e-mail: BOP_351971@mail.ru, http://orcid.org/0000-0002-1779-9125

\section{Author information}

Marina M.Petrova, Dr.Med.Sci., Professor, Prof. V. F. Voino-YasenetskyKrasnoyarsk State Medical University; Address: 1, PartizanZheleznyak Str., Krasnoyarsk, Russian Federation 660022; Phone +7(391)2280628; e-mail: stk99@yandex.ru, http://orcid.org/0000-0002-84930058

Natalya A. Shnayder, Dr.Med.Sci., Prof., St. Petersburg V.M. Bekhterev Psychoneurological Research Institute; Address: 3 Bekhterev St., Saint Petersburg, Russia, 192019; Professor V. F. Voino-YasenetskyKrasnoyarsk State Medical University; Address: 1, Partizan Zheleznyak Str., Krasnoyarsk, Russian Federation 660022; Phone +7(391)22806288; e-mail: NASchnaider@ yandex.ru, http://orcid.org/0000-0002-2840-837X

Elena A. Pronina, Cand.Med.Sci., Associate Professor, Prof. V. F. Voino-Yasenetsky Krasnoyarsk State Medical University; Address: 1, PartizanZheleznyak Str., Krasnoyarsk, Russian Federation 660022; Phone +7(391)2280628; e-mail: diinny@mail.ru, http://orcid. org/0000-0002-8269-6317

Olga P. Bobrova, Cand.Med.Sci., Associate Professor, Prof. V. F. Voino-Yasenetsky Krasnoyarsk State Medical University; Address: 1, PartizanZheleznyak Str., Krasnoyarsk, Russian Federation 660022; Phone +7(391)2280628; e-mail: BOP_351971@mail.ru, http://orcid.org/0000-0002-1779-9125 
This work is licensed under the Creative Commons Attribution 4.0 International License. To view a copy of this license, visit http://creativecommons.org/licenses/by/4.0/. 